\title{
MILLIARCSECOND SCALE VARIABILITY OF A SAMPLE OF SOUTHERN ACTIVE GALACTIC NUCLEI
}

\author{
Faith Hungwe ${ }^{* 1}$ Roopesh Ojha, ${ }^{1,3}$ Roy Booth, ${ }^{1,2}$ Michael Bietenholz, ${ }^{2,5}$ Alan Fey, ${ }^{4}$ \\ Arnoud Collioud ${ }^{6}$ Patrick Charlot $^{6}$ \\ ${ }^{1}$ Rhodes University, P.O Box 94, Grahamstown 6140, South Africa \\ ${ }^{2}$ Hartebeesthoek Radio Astronomy Observatory, P.O Box 443, Krugersdorp, South Africa \\ ${ }^{3}$ NVI/ United States Naval Observatory, 3450 Massachusetts Ave NW, Washington,DC 20392 \\ ${ }^{4}$ United States Naval Observatory, 3450 Massachusetts Ave NW, Washington,DC 20392 \\ ${ }^{5}$ York University, 4700 Keele Street, Toronto, Ontario M3J 1P3 Canada \\ ${ }^{6}$ OASU,Laboratoire d'Astrophysique de Bordeaux (LAB),2 rue de l'Observatoire, B.P. 8933270 \\ FLOIRAC FRANCE \\ E-mail: faith@hartrao.ac.za
}

\begin{abstract}
We present the results of a variability study of Active Galactic Nuclei observed at milliarcsecond resolution using the technique of Very Long Baseline Interferometry. These AGN are currently used to define the ICRF (International Celestial Reference Frame) which is the most accurate reference frame in astronomy, astrometry and geodesy. Among other uses, our analysis will cast light on the suitability of these objects as calibrators for MeerKAT, the South African SKA demonstrator.
\end{abstract}

The 9th European VLBI Network Symposium on The role of VLBI in the Golden Age for Radio Astronomy and EVN Users Meeting

September 23-26, 2008

Bologna, Italy

\footnotetext{
*Speaker.
} 


\section{Introduction}

A major expansion of radio astronomy observing capability is on the way in the Southern Hemisphere with the two SKA precursors, MeerKAT and ASKAP leading to the SKA itself. A significant component of the science goals for these new instruments involve observations with long baselines. For such future observations as well as present southern VLBI observations, it is important to have a dense network of compact calibrator sources whose properties are well known. This presentation of 3 sources out of a sample of 33 southern sources is part of an ongoing pilot program to look at the calibrator properties of southern AGN at milliarcsecond resolution. We use a combination of the variation of core flux density with time, the percentage of total flux density in the core, presence and magnitude of proper motion and the radial extent within which $95 \%$ of the flux density is contained to evaluate the usefulness of these sources at $2.3 \mathrm{GHz}$. No single measure is adequate to pass judgement on the sources but we are developing a way to assign sources into four calibrator categories (A through D) based on their overall properties (see below). This work will be particularly useful for an extended MeerKAT, the South African SKA demonstrator, and the SKA when it becomes operational.

\section{Source Selection, Observations and Data Reduction}

Sources were selected from the United States Naval Observatory Radio Reference Frame Image Database (RRFID). The RRFID is a database of about 4000 images of about 500 sources compiled from geodetic and astrometric VLBI experiments since 1994. Observations were made using the 10 telescopes of NRAO's VLBA, Hartebeesthoek when available, and up to 6 other geodetic antennas across the globe. Observations were made in a dual-frequency bandwidth synthesis mode to facilitate delay measurements for astrometry. This mode of observations also allow imaging at both frequency bands. Eight frequency channels were recorded simultaneously, each $8 \mathrm{MHz}$ wide, with 4 at $\mathrm{S}$-band and 4 at $\mathrm{X}$-band for a total of $32 \mathrm{MHz}$ in each frequency band.

The data were correlated at the Array Operations Center in Socorro, New Mexico. The correlated data was calibrated using NRAO's Astronomical Image Processing System (AIPS). Imaging was done using the CALTECH VLBI imaging software, Difmap. Calculations of superluminal motion were done using a program provided by Glen Piner of Whitier College in California.

\section{Discussion of Individual Sources}

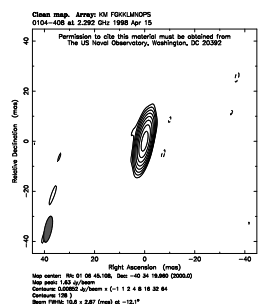

(a)

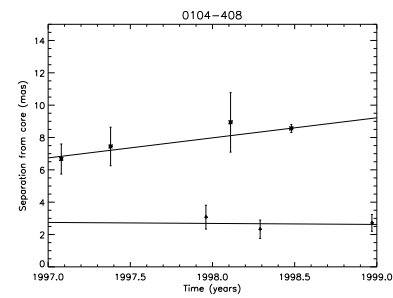

(b)

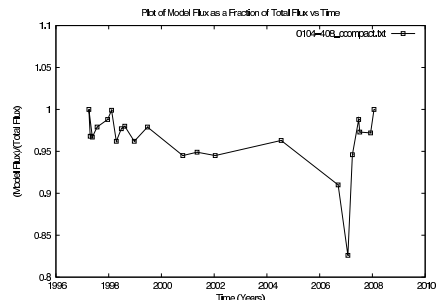

(c)

0104-408: This is essentially a point source with a hint of a component to the east (a). Model fits show two possible components apart from the central core but these components are very stable 
as can be seen from plots of their separation from the core component (b). On average, more than $90 \%$ of the flux density is in the core component (c) and the core flux does not vary significantly. This is a Class A source according to our classification scheme.

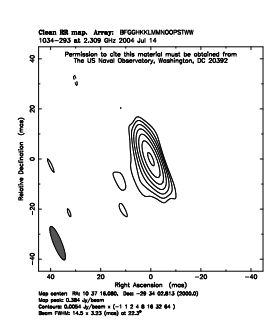

(a)

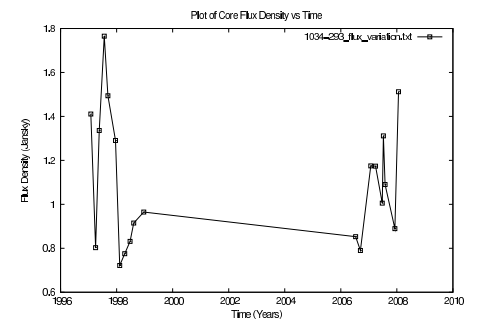

(b)

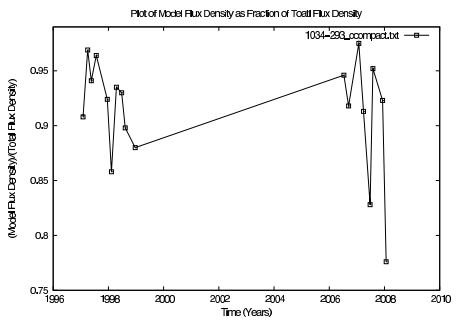

(c)

1034-293: For this source (a), the core flux density varies (b) and model fitting suggests superluminal motion of the inner component with an apparent speed of $(1.96 \pm 0.91) c$ and a speed of $(4.10 \pm 0.99) c$ for the outer component. The core flux density ranges between $85 \%$ and $100 \%$ of the total (c). This is a Class B source.

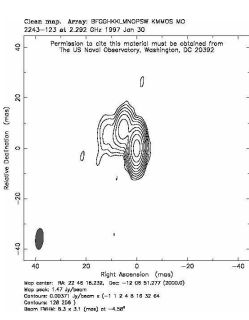

(a)

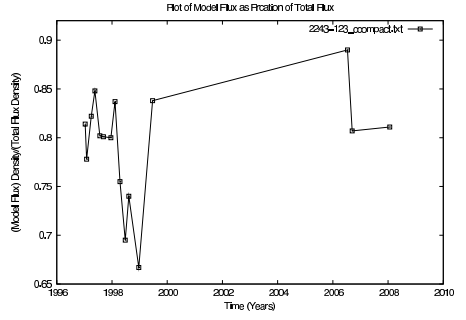

(b)

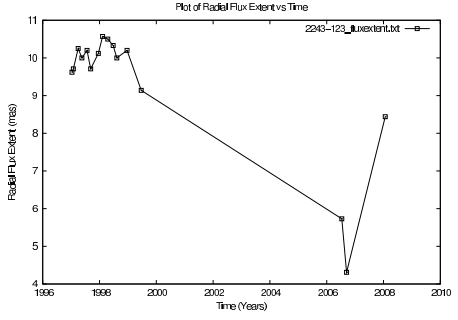

(c)

2243-123: This source is clearly extended (a). Three components were fit. Only about $70 \%$ of the flux density is in the core component (b). The plot of radial extent (c) shows that it decreases as the more distant component fades. A new component is ejected in 2006 after which the radial extent increases again. From our classification scheme, this is a Class $\mathrm{C}$ source.

\section{Conclusion}

We are developing a scheme to classify calibrator sources. This is only a starting point and the classification categories are likely to evolve as we build a more statistically significant sample. We have also measured superluminal motion at $2.3 \mathrm{GHz}$ for some of the sources. We find that both the apparent component locations and speeds at $2.3 \mathrm{GHz}$ do not reproduce well those obtained from the same observing sessions at $8.4 \mathrm{GHz}$.

\section{References}

[1] R. Ojha, A. L. Fey, P. Charlot, et al, VLBI Observations of Southern Hemisphere ICRF Sources. II. Astrometric Suitability Based on Intrinsic Structure,AJ,130, (2529) 2005

[2] B. G. Piner, M. Mahmud, A. L Fey, K. Gospodinova, Relativistic Jets in the Radio Reference Frame Image Database. I. Apparent Speeds from the First 5 Years of Data,AJ,133 (2357) 2007 